\title{
FREMDANAMNESE
}

\section{Zuverlässiger als jeder Alzheimertest}

Für Patienten mit Verdacht auf eine beginnende Alzheimerdemenz gibt es zahlreiche, zum Teil zeitaufwendige Screeningtests. Ein 2-Minuten-Test liefert ein genauso zuverlässiges Ergebnis. Dabei wird nicht der Patient selbst befragt, sondern ein Familienangehöriger oder enger Freund. In einem kurzen Fragebogen (Ascertain Dementia, AD8) geben die Angehörigen darüber Auskunft, ob der Pati- ent z. B. neuerdings Probleme hat, eine neue Fernsehfernbedienung zu benutzen, ob er Fragen und Geschichten wiederholt, ob er sich Verabredungen nicht mehr merken kann und ob sein Interesse an Hobbys nachgelassen hat. Werden zwei oder mehr Fragen mit ,ja“ beantwortet, besteht der Verdacht auf Alzheimer und der Patient wird einer weitergehenden Diagnostik zugeführt.

Brain, 2010; DOl: 10.1093/brain/awq204

\section{BISPHOSPHONATE}

\section{Erhöhtes Risiko für Ösophaguskarzinome?}

Die Anwendung von oralen Bisphosphonaten kann einer Fall-Kontroll-Studie zufolge das Risiko für ein Ösophaguskarzinom erhöhen. Nach fünf Jahren Therapie hatten doppelt so viele Patienten ein Ösophaguskarzinom wie Personen ohne eine solche Behandlung. Eine Korrelation mit Magen- oder kolorektalen Karzinomen bestand dagegen nicht. Wegen des möglichen Zusammenhangs mit malignen Ver-

\section{US-STUDIE}

\section{Luftverschmutzung schuld an Diabetes?}

Einen engen Zusammenhang zwischen Luftverschmutzung und Diabetesinzidenz wollen Forscher aus Boston herausgefunden haben. Demnach sind Feinstaubpartikel von 0,1-2,5 $\mathrm{nm}$ Durchmesser in der Lage, Diabetes zu induzieren. Nimmt die Menge der sog. PM-2,5-Partikel um $10 \mu \mathrm{g} /$ $\mathrm{m}^{3} \mathrm{zu}$, steigt die Diabetesinzidenz um 1\% an. Verglich man Regionen mit der niedrigsten und der höchsten Feinstaubbelastung, differierte die Diabetesinzidenz um 20\%. PM-2,5-Partikel werden durch Autoabgase und Industriedunst freigesetzt. Diabetes Care 2010; 33:2196; DOI: 10.2337/dc10-0698 änderungen der Speiseröhre ist es besonders wichtig, dass die Patienten die Einnahmevorschriften für Bisphosphonate einhalten: Die Tabletten sollten nach dem Aufstehen auf nüchternen Magen mit einem ganzen Glas Leitungswasser eingenommen werden, und der Patient sollte mindestens weitere 30 Minuten nüchtern bleiben und sich nicht hinlegen.

BMJ 2010;341:C4444; doi:10.1136/bmj.c4444

\section{CAVE EXZESSIVE ZUNAHME}

\section{Dicke Schwangere, dickes Baby}

Das Geburtsgewicht von Kindern ist eng korreliert mit der Gewichtszunahme der Mutter in der Schwangerschaft. Nimmt die Schwangere sogar mehr als $24 \mathrm{~kg} \mathrm{zu}$, verdoppelt sich die Wahrscheinlichkeit, dass ihr Neugeborenes mehr als $4 \mathrm{~kg}$ auf die Waage bringt. Dieser Zusammenhang ist unabhängig von genetischen Faktoren, denn er lässt sich auch dann beobachten, wenn eine Frau mehrere Schwangerschaften mit unterschiedlichem Gewichtsverlauf hat.

Lancet 2010;376:984-90

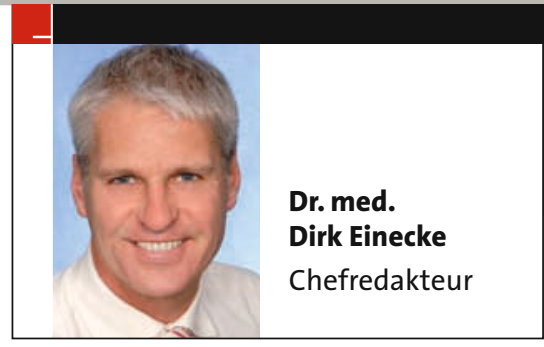

AKUPUNKTUR NACH APOPLEX Pikser ohne Wirkung

Die Erholung von Schlaganfallpatienten lässt sich durch Akupunktur nicht beeinflussen. Das ist das Fazit eines systematischen Reviews, in dem Vergleichsstudien von Akupunktur und Scheinakupunktur ausgewertet wurden. In sieben Studien wurde im akuten und subakuten Stadium nach dem Schlaganfall genadelt - insgesamt ohne signifikanten Unterschied zwischen Schein- und Verumtherapie in Bezug auf funktionellen Status und Alltagsaktivitäten. Die Akupunktur zu einem späteren Zeitpunkt blieb in jeder von drei weiteren Studien ebenfalls wirkungslos. CMAJ, 27. September 2010; DOI:10.1503/cmaj.o91113

\section{ENURESIS}

\section{Rückfall bei Schulbeginn}

Im September, wenn das Schuljahr wieder anfängt, sehen Urologen mehr Kinder mit Enuresis. Das Bettnässen hat in der Mehrzahl der Fälle keine organischen Ursachen. Wahrscheinlicher sind zwei andere Ursachen für die Häufung der Enuresisfälle: Erstens bedeutet der Schulbeginn einen emotionalen Stress, der Bettnässen begünstigt, und zweitens unterscheidet sich der Lebensrhythmus - und damit auch die Toilettengewohnheiten - dieser Kinder in den Ferien und während der Schulzeit erheblich. Man sollte die Kinder daher in den letzten Ferientagen auf eine schulkompatible Schlafenszeit und Flüssigkeitsaufnahme umstellen.

Johns Hopkins Medicine, 27. September 2010 